NISSUNA UMANA INVESTIGAZIONE SI PUO DIMANDARE VERA SCIENZIA S'ESSA NON PASSA PER LE MATEMATICHE DIMOSTRAZIONI LEONARDO DA VINCI

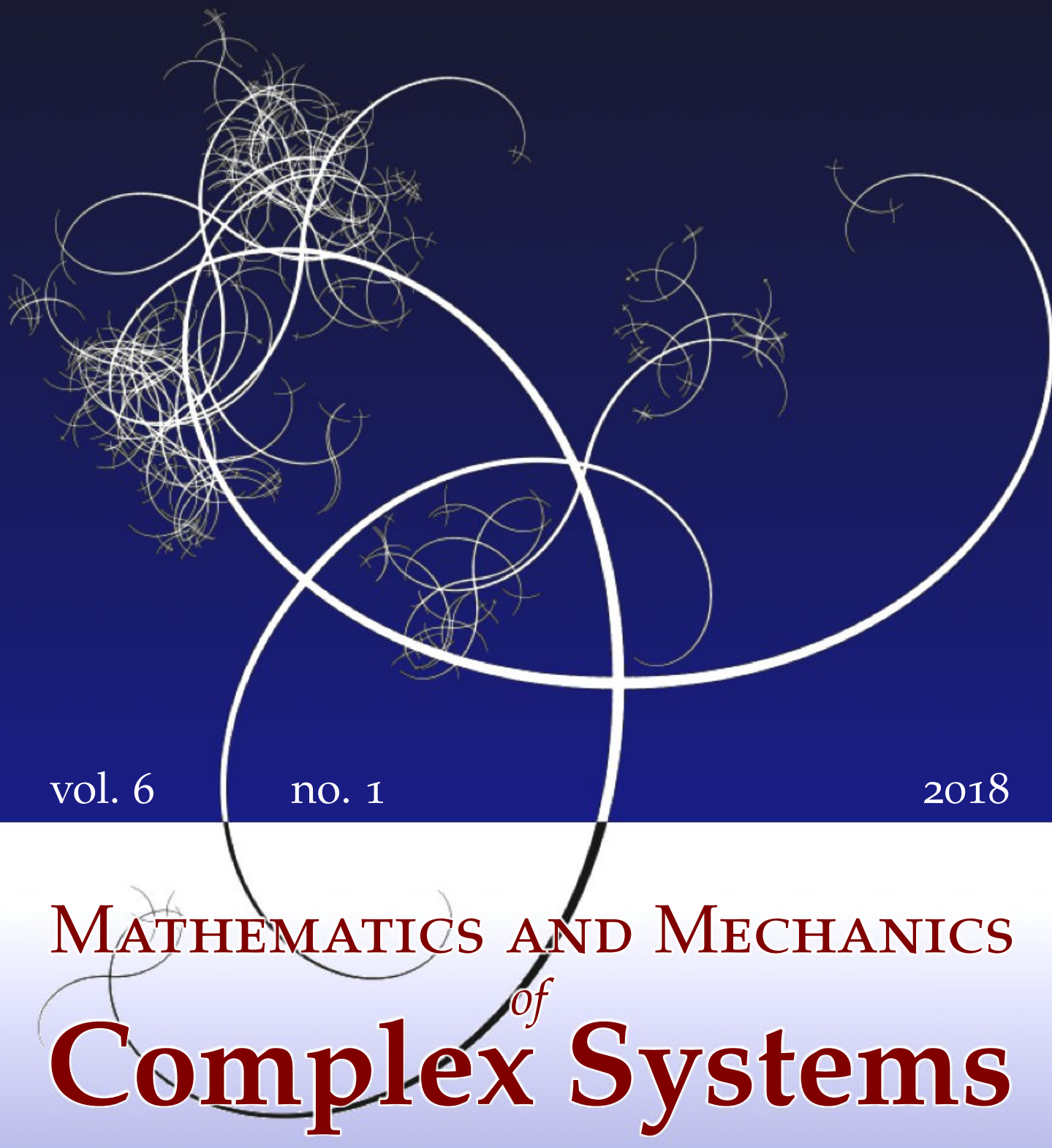

DANIELE PUGLISI

OPERATORS ON BOURGAIN-DELBAEN SPACES 


\title{
OPERATORS ON BOURGAIN-DELBAEN SPACES
}

\author{
DANIELE PUGLISI
}

We prove that, whenever we pick real numbers $a, b$ such that $0<b<a<1$, $a+b>1$, and $a^{3}+b^{3}=1$, then every bounded linear operator from $\ell_{2}$ to $X_{a, b}$ and from $X_{a, b}$ to $\ell_{2}$ must be compact, where $X_{a, b}$ is the Bourgain-Delbaen space.

\section{Introduction}

This note concerns a question posed to us by R. Aron during the conference Function Theory on Infinite Dimensional Spaces XIV (Madrid, 2016). We were told that the same question was raised during some other conference in 1987 and it was solved by J. Bourgain soon after. Since at that time J. Bourgain decided not to publish it, the result remains only announced in [Alencar et al. 1987].

Throughout this note we shall use standard notation. For Banach spaces $X, Y$ we denote by $\mathscr{L}(X, Y)$ the space of all bounded linear operators from $X$ to $Y$ and by $\mathscr{K}(X, Y)$ its ideal of compact operators. It is a classical result due to Pitt [1936] that every linear continuous operator from $\ell_{p}$ to $\ell_{q}$ must be compact for all $q<p$. It is worth noting here that this result has been generalized in [Puglisi 2014, Lemma 3.5] where $\ell_{p}$-direct sums of sequences of suitable Banach spaces have been used. Following Pitt's theorem, in [Alencar et al. 1987, Lemma 5], the following was proved.

Proposition 1.1. Suppose a Banach space $X$ has the property that, for some $p>1$, $\mathscr{L}\left(X, \ell_{p}\right)=\mathscr{K}\left(X, \ell_{p}\right)$. Then $\mathscr{L}\left(X, \ell_{q}\right)=\mathscr{K}\left(X, \ell_{q}\right)$ for all $1<q<p$.

This proposition shows that there are nontrivial examples of triples $(X, Y, Z)$ of Banach spaces with the property that, if every continuous linear operator from $X$ to $Y$ is compact and every continuous linear operator from $Y$ to $Z$ is compact, then every continuous linear operator from $X$ to $Z$ is compact. In case this happens, we say that the triple $(X, Y, Z)$ has the compact operators transitivity property. Therefore, it is quite natural to raise the following:

\section{Communicated by Raffaele Esposito.}

The author was supported by the "National Group for Algebraic and Geometric Structures, and their Applications" (GNSAGA, INdAM).

MSC2010: 47B10.

Keywords: compact operators, Bourgain-Delbaen spaces. 
Question (R. Aron, 1987). Does every triple $(X, Y, Z)$ of Banach spaces have the compact operators transitivity property?

As announced in [Alencar et al. 1987], an answer was given by J. Bourgain but unpublished. In this note, we give a simple counterexample to the above question. It is also worth recalling the example given in [Argyros and Haydon 2011], where, if we denote by $\mathfrak{X}_{\mathrm{AH}}(L)$ the Argyros-Haydon space constructed on the infinite subset $L \subseteq \mathbb{N}$, the following spectacular result holds.

Theorem 1.2. If $L \cap L^{\prime}$ is finite, then every bounded linear operator from $\mathfrak{X}_{\mathrm{AH}}(L)$ to $\mathfrak{X}_{\mathrm{AH}}\left(L^{\prime}\right)$ must be compact.

In any case, the example we propose is much simpler.

\section{The Bourgain-Delbaen space}

In this section we would like to briefly review the construction of the classical Bourgain-Delbaen space $X_{a, b}$ [1980] using the original notation, even if we recommend the reader the Ph.D. thesis of M. Tarbard [2012], where the exposition is very clear.

Definition 2.1. If $X$ and $Y$ are Banach spaces, then the Banach-Mazur distance between $X$ and $Y$ is given by

$$
d(X, Y)=\inf \left\{\|T\| \cdot\left\|T^{-1}\right\| \mid T: X \rightarrow Y \text { is an onto isomorphism }\right\} .
$$

Definition 2.2. (i) A Banach space $X$ is an $\mathscr{L}_{\infty, \lambda}$ space $(\lambda \geq 1)$ if, for all $E \subseteq X$, where $E$ is a finite-dimensional subspace of $X$, there is a finite-dimensional subspace $F \subseteq X$, such that $E \subseteq F$ and $d\left(F, \ell_{\infty}^{\operatorname{dim} F}\right) \leq \lambda$. Here $\ell_{\infty}^{n}$ means the $n$-dimensional real vector space $\mathbb{R}^{n}$ endowed with the norm $\left\|\left(x_{1}, \ldots, x_{n}\right)\right\|=$ $\max _{1 \leq k \leq n}\left|x_{k}\right|$.

(ii) If $X$ is an $\mathscr{L}_{\infty, \lambda}$ space for some $\lambda$, then we simply say that $X$ is an $\mathscr{L}_{\infty}$ space.

For a $\lambda>1$ let us fix from now on real numbers $a, b$ such that

(1) $0<b<a<1$,

(2) $a+2 b \lambda \leq \lambda$,

(3) $a+b>1$.

By induction, first we construct a sequence $\left(d_{n}\right)_{n} \subseteq \mathbb{R}$ and

$$
i_{m, l}: \operatorname{span}\left\{e_{i} \mid 1 \leq i \leq d_{m}\right\} \rightarrow \operatorname{span}\left\{e_{i} \mid 1 \leq i \leq d_{l}\right\}
$$

with $m<l \leq n$, where $e_{i}$ is the standard basic sequence, i.e., $e_{i}(j)=\delta_{i, j}$. Let us denote $E_{n}=\operatorname{span}\left\{e_{i} \mid 1 \leq i \leq d_{n}\right\} \subseteq \ell_{\infty}$.

Let $d_{1}=1$. Suppose $d_{1}, \ldots, d_{n}$ are known as well as $\left\{i_{m, l} \mid 1 \leq m<l \leq n\right\}$ satisfying 
(a) $\pi_{m} \circ i_{m, n}=\operatorname{id}_{\operatorname{span}\left\{e_{i} \mid 1 \leq i \leq d_{m}\right\}}$ for $m<n$,

(b) $i_{l, n} \circ i_{m, l}=i_{m, n}$ for $m<l<n$,

where $\pi_{m}$ is the natural projection to the first $d_{m}$ coordinates. For all

$$
\gamma=\left(m, i, j, \varepsilon^{\prime}, \varepsilon^{\prime \prime}\right) \quad \text { with } m<n, 1 \leq i<d_{m}, 1 \leq j \leq d_{n} \text {, and } \varepsilon^{\prime}, \varepsilon^{\prime \prime}= \pm 1,
$$

let us define a functional in $\left(\operatorname{span}\left\{e_{i} \mid 1 \leq i \leq d_{n}\right\}\right)^{*}$ as

$$
c_{\gamma}^{*}(x)=\varepsilon^{\prime} a x_{i}+\varepsilon^{\prime \prime} b\left(x-i_{m, n} \circ \pi_{m}(x)\right)_{j} .
$$

Let us consider the set of all such functionals, namely

$\mathscr{F}_{n}=\left\{c_{\gamma}^{*} \mid \gamma=\left(m, i, j, \varepsilon^{\prime}, \varepsilon^{\prime \prime}\right), m<n, 1 \leq i<d_{m}, 1 \leq j \leq d_{n}\right.$, and $\left.\varepsilon^{\prime}, \varepsilon^{\prime \prime}= \pm 1\right\} ;$ then we define

$$
d_{n+1}=d_{n}+\operatorname{card} \mathscr{F}_{n}
$$

and

$$
i_{n, n+1}(x):=(\underbrace{x_{1}, \ldots, x_{d_{n}},\left(c_{\gamma}^{*}(x)\right)_{\gamma \in \mathscr{F}_{n}}}_{d_{n+1}}, 0,0, \ldots, 0, \ldots) .
$$

For each $n \in \mathbb{N}$ let us consider

$$
i_{n}: \operatorname{span}\left\{e_{i} \mid 1 \leq i \leq d_{n}\right\} \rightarrow \ell_{\infty}
$$

defined as

$$
i_{n}=\lim _{m \rightarrow \infty}\left(i_{n, n+1} \circ i_{n+1, n+2} \circ \cdots \circ i_{m-1, m}\right) .
$$

It has been shown by J. Bourgain and F. Delbaen [1980, Lemma 4.2(8)] that $\left\|i_{n}\right\| \leq \lambda$ for all $n \in \mathbb{N}$. Therefore, if we denote

$$
F_{n}=i_{n}\left(\operatorname{span}\left\{e_{i} \mid 1 \leq i \leq d_{n}\right\}\right),
$$

the space of Bourgain-Delbaen $X_{a, b}$ is defined as the closure in $\ell_{\infty}$ of the countable union

$$
\bigcup_{n \in \mathbb{N}} F_{n}
$$

Since $\pi_{n} \circ i_{n}=\operatorname{id}_{E_{n}}$, we have that $d\left(F_{n}, \ell_{\infty}^{d_{n}}\right)=d\left(F_{n}, E_{n}\right) \leq\left\|\pi_{n}\right\| \cdot\left\|i_{n}\right\| \leq \lambda$. From [Lindenstrauss and Tzafriri 1973], since $X_{a, b}$ is the closure of an increasing sequence of finite-dimensional subspaces $F_{n}$ with $d\left(F_{n}, \ell_{\infty}^{d_{n}}\right) \leq \lambda$, then

( $\alpha) X_{a, b}$ is an $\mathscr{L}_{\infty, \lambda+\varepsilon}$ space for all $\varepsilon>0$.

Curiously, the space $X_{a, b}$ satisfies the following properties, which make the space very interesting:

( $\beta$ ) $X_{a, b}$ does not contain any copy of $\ell_{1}$; since $X_{a, b}$ is an $\mathscr{L}_{\infty}$ space, it follows from a result of Lewis and Stegall [1973] that $X_{a, b}^{*}$ is isomorphic to $\ell_{1}$; 
( $\gamma) X_{a, b}$ has the Radon-Nikodym property [Bourgain and Delbaen 1980, Lemma 5.4];

( $\delta) X_{a, b}$ is reflexive saturated [Bourgain and Delbaen 1980, Lemma 5.5] (i.e., every infinite-dimensional closed subspace contains an infinite-dimensional reflexive subspace).

Before closing this section, we would like to review a final peculiar property. Let $\alpha$ be the unique number such that

$$
a^{1 /(1-\alpha)}+b^{1 /(1-\alpha)}=1 .
$$

Then in $X_{a, b}$ the following holds. For a proof see [Bourgain and Delbaen 1980, Lemma 5.9] (see also [Haydon 2000]).

Proposition 2.3. For every sequence $\left(x_{n}\right)_{n} \subseteq X_{a, b}$ such that

(i) $\left\|x_{n}\right\|=1$ for all $n \in \mathbb{N}$,

(ii) $x_{n} \rightarrow 0$ weakly,

there exist $C>0$ and a subsequence $\left(x_{k_{n}}\right)_{n}$ such that

$$
\left\|x_{k_{1}}+\cdots+x_{k_{n}}\right\| \geq C n^{\alpha} \quad \text { for all } n \in \mathbb{N} .
$$

\section{Operators on the space $X_{a, b}$}

Throughout this section we fix $a, b$ such that

(i) $0<b<a<1$,

(ii) $a+b>1$,

(iii) $a^{3}+b^{3}=1$.

Before stating the main result, we would like to recall a well known result. Since the proof is easy, we include it for the sake of completeness.

Proposition 3.1. Let $X$ be a Banach space with a Schauder basis and $Y$ any Banach space, and let $T: X \rightarrow Y$ be a bounded linear operator. If

$$
\left\|T\left(x_{n}\right)\right\| \stackrel{n \rightarrow \infty}{\longrightarrow} 0 \text { for all bounded block basic sequences }\left(x_{n}\right)_{n},
$$

then $T$ is compact.

Proof. Let us denote by $\left(x_{n}\right)_{n}$ a Schauder basis of $X$ with basis constant $K$, and let us denote by

$$
P_{n}: X \rightarrow X
$$

the $n$-th projection associated to the basis; i.e.,

$$
P_{n}\left(\sum_{i=1}^{\infty} a_{i} x_{i}\right)=\sum_{i=1}^{n} a_{i} x_{i} .
$$


We show that

$$
\left\|T-T \circ P_{n}\right\| \stackrel{n \rightarrow \infty}{\longrightarrow} 0 ;
$$

i.e., $T$ is the uniform limit of finite rank operators.

Let us assume we are not in this situation; that means there exist $\delta>0$, a strictly increasing sequence of natural numbers $\left(k_{n}\right)_{n}$, and a sequence of norm-1 $\left(z_{n}\right)_{n} \subseteq X$ such that

$$
\left\|\left(T-T \circ P_{k_{n}}\right)\left(z_{n}\right)\right\|>\delta .
$$

Since $z_{1}=\lim _{n} P_{n}\left(z_{1}\right)$, we can find $s_{1}>k_{1}$ such that

$$
\left\|z_{1}-P_{S_{1}}\left(z_{1}\right)\right\|<\frac{\delta}{2\|T\|} .
$$

Let us define $y_{1}=P_{s_{1}}\left(z_{1}\right)-P_{k_{1}}\left(z_{1}\right)$. Then

$$
\begin{aligned}
\left\|y_{1}\right\| \leq 2 K \quad \text { and } \quad\left\|T\left(y_{1}\right)\right\| & \geq\left\|\left(T-T \circ P_{k_{1}}\right)\left(z_{1}\right)\right\|-\left\|\left(T-T \circ P_{S_{1}}\right)\left(z_{1}\right)\right\| \\
& >\frac{\delta}{2} .
\end{aligned}
$$

Let $k_{j_{2}}>s_{1}$. Similarly, we find $s_{2}>k_{j_{2}}$ such that

$$
\left\|z_{j_{2}}-P_{s_{2}}\left(z_{j_{2}}\right)\right\|<\frac{\delta}{2\|T\|} .
$$

Let us define $y_{2}=P_{s_{2}}\left(z_{j_{2}}\right)-P_{k_{j_{2}}}\left(z_{j_{2}}\right)$. Then

$$
\left\|y_{2}\right\| \leq 2 K \quad \text { and } \quad\left\|T\left(y_{2}\right)\right\|>\frac{\delta}{2} .
$$

Iterating this process we find a bounded block basic sequence $\left(y_{n}\right)_{n}$ of $Y$ such that $\left\|T\left(y_{n}\right)\right\|>\delta / 2$, against the assumption (2).

Theorem 3.2. Every bounded linear operator from $\ell_{2}$ to $X_{a, b}$ and from $X_{a, b}$ to $\ell_{2}$ must be compact.

Proof. Since $X_{a, b}^{*}$ has the Schur property, it is obvious that any operator $X_{a, b}$ to $\ell_{2}$ is compact.

Suppose there is a bounded linear operator

$$
T: \ell_{2} \rightarrow X_{a, b}
$$

which is not compact. By the previous proposition, there must exist a bounded block basic sequence $\left(x_{n}\right)_{n} \subseteq \ell_{2}$ such that

$$
\left\|T\left(x_{n}\right)\right\|>\delta \quad \text { for some } \delta>0 .
$$

It is worth recalling that a basis in a Banach space is shrinking if and only if any bounded block basic sequence is weakly null. Since the basis in $\ell_{2}$ is shrinking, 
we have that the sequence $\left(x_{n}\right)_{n}$ is weakly null (and so is $\left.\left(T\left(x_{n}\right)\right)_{n}\right)$. Therefore, the sequence

$$
\left(\frac{T\left(x_{n}\right)}{\left\|T\left(x_{n}\right)\right\|}\right)_{n} \text { is weakly null. }
$$

By Proposition 2.3 and assumption (iii) above, by passing to a subsequence, there exists $C>0$ such that

$$
\left\|\frac{T\left(x_{1}\right)}{\left\|T\left(x_{1}\right)\right\|}+\cdots+\frac{T\left(x_{n}\right)}{\left\|T\left(x_{n}\right)\right\|}\right\| \geq C n^{2 / 3} .
$$

On the other hand, since bounded block basic sequences in $\ell_{2}$ are unconditionally basic sequences (and so is $\left.\left(T\left(x_{n}\right)\right)_{n}\right)$, it also follows that

$$
\left\|T\left(x_{1}\right)+\cdots+T\left(x_{n}\right)\right\| \geq C^{\prime} n^{2 / 3}
$$

for some $C^{\prime}>0$. Therefore,

$$
\begin{aligned}
C^{\prime} n^{2 / 3} & \leq\left\|T\left(x_{1}\right)+\cdots+T\left(x_{n}\right)\right\| \\
& \leq\|T\|\left\|x_{1}+\cdots+x_{n}\right\|_{\ell_{2}} \\
& \leq\|T\| C^{\prime \prime} n^{1 / 2},
\end{aligned}
$$

where the last inequality follows from the fact that $\left(x_{n}\right)_{n}$ is a bounded block sequence in $\ell_{2}$. This would imply that

$$
n^{1 / 6} \leq\|T\| \frac{C^{\prime \prime}}{C^{\prime}} \quad \text { for all } n \in \mathbb{N},
$$

which is a contradiction. This completes the proof.

We would like to finish this note with the following natural questions.

Question. (i) Does there exist a pair of Banach spaces $(X, Y)$ such that every $n$-homogeneous polynomial from $X$ to $Y$ and from $Y$ to $X$ must be compact?

(ii) Let $Y$ and $Z$ be reflexive Banach spaces. Does, for every Banach space $X$, the triple $(X, Y, Z)$ have the compact operators transitivity property?

\section{Acknowledgement}

We wish to thank R. Aron for bringing to our attention the question.

\section{References}

[Alencar et al. 1987] R. Alencar, R. M. Aron, and G. Fricke, “Tensor products of Tsirelson's space”, Illinois J. Math. 31:1 (1987), 17-23.

[Argyros and Haydon 2011] S. A. Argyros and R. G. Haydon, "A hereditarily indecomposable $\mathscr{L}_{\infty}$ space that solves the scalar-plus-compact problem", Acta Math. 206:1 (2011), 1-54. 
[Bourgain and Delbaen 1980] J. Bourgain and F. Delbaen, "A class of special $\mathscr{L}_{\infty}$ spaces", Acta Math. 145:3-4 (1980), 155-176.

[Haydon 2000] R. Haydon, "Subspaces of the Bourgain-Delbaen space", Studia Math. 139:3 (2000), 275-293.

[Lewis and Stegall 1973] D. R. Lewis and C. Stegall, "Banach spaces whose duals are isomorphic to $l_{1}(\Gamma)$ ", J. Functional Analysis 12 (1973), 177-187.

[Lindenstrauss and Tzafriri 1973] J. Lindenstrauss and L. Tzafriri, Classical Banach spaces, Lecture Notes in Mathematics 338, Springer, 1973.

[Pitt 1936] H. R. Pitt, “A note on bilinear forms”, J. London Math. Soc. 11:3 (1936), 174-180.

[Puglisi 2014] D. Puglisi, "The position of $\mathscr{K}(X, Y)$ in $\mathscr{L}(X, Y)$ ", Glasg. Math. J. 56:2 (2014), 409417.

[Tarbard 2012] M. Tarbard, Operators on Banach spaces of Bourgain-Delbaen type, Ph.D. thesis, University of Oxford, 2012. arXiv

Received 20 Oct 2017. Revised 11 Dec 2017. Accepted 11 Jan 2018.

DANIELE PUGLISI: dpuglisi@dmi.unict.it

Department of Mathematics and Computer Sciences, University of Catania, Catania, Italy

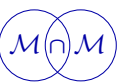

